\title{
Monitoreo mediante Emisión Acústica de vigas de hormigón de alta resistencia con y sin fibras expuesto a alta temperatura
}

\author{
Acoustic Emission monitoring of high-strength \\ concrete beams with and without fibers \\ exposed to high temperature
}

Hernán Xargay ${ }^{1,2}$, Paula Folino ${ }^{2}$, Nicolás Nuñez ${ }^{1}$, Martín Gómez ${ }^{1}$

\footnotetext{
${ }^{1}$ ICES-Grupo de Ondas Elásticas, CAC, CNEA, Av. Gral Paz 1499, San Martín, Buenos Aires, Argentina.

${ }^{2}$ Universidad de Buenos Aires, Facultad de Ingeniería, LMNI, INTECIN (UBA-CONICET), Av. Gral. Las Heras 2214, Buenos Aires, Buenos Aires, Argentina.

e-mail: hernanxargay@cnea.gov.ar

e-mail: pfolino@fi.uba.ar; nnunez@cnea.gov.ar; mpgomez@cnea.gov.ar
}

\begin{abstract}
RESUMEN
La adición de fibras a la matriz cementicia, dando lugar a los hormigones reforzados con fibras, tiene por objeto mejorar la baja ductilidad que se observa en la respuesta mecánica del hormigón frente a distintos tipos de solicitación, particularmente bajo esfuerzos de tracción directa. En este trabajo, se estudia el proceso de fisuración y degradación mecánica de elementos de hormigón utilizando la técnica de emisión acústica, con el objetivo de analizar la incidencia de la acción de altas temperaturas sobre el comportamiento de falla de vigas de hormigón de alta resistencia con y sin fibras. Se presentan resultados de un plan experimental que incluye distintos ensayos mecánicos sobre especímenes de hormigón con y sin adición de una combinación híbrida de fibras de acero y de polipropileno. La incorporación de las fibras de polipropileno tiene por objeto mejorar el comportamiento del hormigón frente a temperaturas elevadas. Se analiza la evolución de las emisiones acústicas registradas durante los ensayos de flexión y su relación con la respuesta mecánica. Se observa que frente a la exposición a una temperatura de $600^{\circ} \mathrm{C}$, las propiedades del hormigón reforzado con fibras fueron relativamente menos afectadas que las del hormigón sin fibras, conservando cierta capacidad de absorber energía en el pos-pico. Asimismo, se confirma que la técnica de monitoreo utilizada tiene una correlación directa con el proceso de degradación del hormigón.
\end{abstract}

Palabras clave: Hormigón de alta resistencia, Hormigón reforzado con fibras, Emisión Acústica, Alta temperatura, Ensayo de flexión de tres puntos.

\section{ABSTRACT}

The addition of fibers to the cementitious matrix, giving rise to fiber-reinforced concrete, aims to improve the low ductility observed in concrete mechanical response under different load scenarios, particularly under direct tension. In this work, cracking and mechanical degradation processes of concrete elements using acoustic emission technique are examined, with the objective of analyzing the incidence of high temperatures on the failure behavior of high-strength concrete beams with and without fibers. Results of an experimental campaign including different mechanical tests on concrete specimens with and without the addition of a hybrid combination of steel and polypropylene fibers are presented. The incorporation of polypropylene fibers is intended to improve the behavior of concrete against elevated temperatures. The evolution of acoustic emission measurements during bending tests and its relation with the mechanical response is analyzed. It is observed that after being exposed to a temperature of $600^{\circ} \mathrm{C}$, properties of fiber reinforced concrete were relatively less affected than those of plain concrete, retaining some capacity to absorb energy in the post-peak regime. It is also confirmed that the monitoring technique used, has a direct correlation with the degradation process of concrete.

Keywords: High-strength concrete, Fiber-reinforced concrete, Acoustic emission, High temperature, Threepoint-bending test. 


\section{INTRODUCCIÓN}

En las últimas décadas se ha extendido considerablemente la utilización de Hormigones de Alta Resistencia (HSC, High-Strength Concrete) en la industria de la construcción. El incremento de la resistencia a compresión es posible mediante la reducción de la relación agua-cemento incorporando aditivos químicos y también adiciones minerales. Su microestructura con menor permeabilidad y porosidad, mejora la durabilidad frente a las acciones nocivas del medio ambiente en que se encuentra, redundando en una mayor vida útil en servicio. Sin embargo, el aumento de la resistencia a la compresión implica un comportamiento mecánico menos dúctil [1]. La adición de cantidades entre el $0,5 \%$ y $1 \%$ en volumen de fibras de acero en el hormigón durante el mezclado, permite incrementar la tenacidad y limitar tanto el ancho como la propagación de las fisuras [2]. Frente a la acción de altas temperaturas, debido a la mayor proporción de pasta de cemento y microestructura más densa que los hormigones convencionales o de baja resistencia (NSC, Normal Strength Concrete), se generan importantes gradientes internos de presión de vapor de agua que provocan microfisuración en la matriz cementicia y pueden derivar en el desprendimiento explosivo de partes paralelas a la superficie calentada. Este fenómeno se denomina "spalling", y aunque también se produce en los NSCs, es más acentuado en los HSCs. Se ha demostrado que la incorporación de pequeñas cantidades de microfibras de polipropileno puede mejorar parcialmente este efecto ya que este material se funde a temperaturas del orden de $160^{\circ} \mathrm{C}$, originando cavidades que permiten una reducción de las presiones de vapor [3].

Históricamente, el estudio del hormigón bajo la acción de alta temperatura fue motivado por dos tópicos: la resistencia al fuego de túneles y edificios y el comportamiento de contenedores de reactores nucleares. El diseño de nuevos hormigones demanda conocer su comportamiento bajo diversas condiciones ambientales, entre ellas para temperaturas entre $20^{\circ} \mathrm{C}$ y $750^{\circ} \mathrm{C}$ [4].

El hormigón expuesto a temperatura sufre cambios químicos y físicos como pérdida del contenido de humedad de su matriz, modificación de la microestructura y descomposición de los agregados [5]. Los principales efectos varían con el nivel de temperatura alcanzado. Hasta los $200^{\circ} \mathrm{C}$ no se observan cambios significativos en las propiedades mecánicas del material debido a que se produce la evaporación del agua libre y adsorbida y su expulsión a través de la red de poros interconectados. A pesar de ello, pueden generarse daños estructurales debidos a la acción de gradientes térmicos y cambios de algunas propiedades físicas del material, $\mathrm{y}$, en consecuencia, la durabilidad podría verse comprometida [6,7]. Con el aumento de la temperatura y hasta los $500^{\circ} \mathrm{C}$, crece el tamaño promedio de los poros debido a la pérdida de agua y deshidratación de los silicatos de calcio hidratados (C-S-H) [8]. Alcanzando los $450^{\circ} \mathrm{C}$, el hidróxido de calcio $\left(\mathrm{Ca}(\mathrm{OH})_{2}\right)$ de la pasta de cemento se descompone en óxido de calcio $\left(\mathrm{CaO}_{2}\right)$ y agua. Hasta estas temperaturas los agregados se encuentran más o menos estables, excepto los de origen silicio. A partir de los $500^{\circ} \mathrm{C}$ los cambios químicos y físicos del hormigón se tornan muy importantes e irreversibles. Cerca de $\operatorname{los} 600^{\circ} \mathrm{C}$, se produce la transformación de fase cristalina $\alpha$ a $\beta$ del cuarzo de los agregados, generando expansiones deletéreas. A partir de los $600^{\circ} \mathrm{C}$, comienza la descomposición química del C-S-H que constituye el principal compuesto resistente de la matriz cementicia. Entre $600^{\circ} \mathrm{C}$ y $800^{\circ} \mathrm{C}$ el carbonato de calcio $\left(\mathrm{CaCO}_{3}\right)$ se disocia y luego de los $800^{\circ} \mathrm{C}$ ya se ha perdido toda el agua de hidratación o químicamente combinada, siendo la capacidad resistente muy baja. Finalmente, a los $1200^{\circ} \mathrm{C}$ comienza a fundirse [9].

Desde el punto de vista de las propiedades mecánicas, diversas investigaciones han mostrado que la acción térmica genera modificaciones sustanciales en la cohesión y resistencia del hormigón, degradando el módulo de Young y el coeficiente de Poisson [1,5, 10, 11, 12]. El hormigón se comporta de manera más dúctil aumentando la deformación correspondiente al pico de resistencia a compresión a medida que se eleva la temperatura. Además, se ha observado un incremento de la energía de fractura liberada hasta los $400^{\circ} \mathrm{C}$ debido a que las superficies donde se localiza la falla son más tortuosas. Para mayores temperaturas disminuye por el excesivo daño térmico y la fuerte pérdida de resistencia a tracción y a compresión [13,14].

El comportamiento del hormigón sometido a alta temperatura es sensible a muchos factores ambientales como: tasa de calentamiento, tiempo de permanencia de la temperatura, forma de enfriamiento y nivel de humedad, entre otros [9].

La técnica de la Emisión Acústica (AE, Acoustic Emission) ha sido utilizada para monitorear en tiempo real ensayos mecánicos de especímenes de hormigón debido a su gran sensibilidad para detectar la evolución del daño desde su comienzo [15-18]. La AE es un fenómeno físico que ocurre dentro de los materiales cuando son solicitados. Habitualmente se la define como la liberación espontánea de energía elástica en la forma de ondas elásticas transitorias [19]. La aplicación de cargas en un elemento produce modificaciones estructurales internas como el crecimiento de fisuras, deformación plástica y fricción, las cuales suelen estar acompañadas por la emisión de ondas elásticas. Éstas contienen información sobre el comportamiento interno 
del material. Las ondas se propagan desde sus fuentes hasta la superficie donde excitan a sensores piezoeléctricos que las convierten en una señal eléctrica. Estas señales son posteriormente procesadas a través de un equipamiento electrónico adecuado y se obtienen datos característicos potencialmente utilizables para detectar, localizar e inferir el origen de las fuentes. Usualmente, la AE en el hormigón es registrada en una banda de frecuencias entre $20 \mathrm{kHz}$ y $300 \mathrm{kHz}$, es decir, en el rango del ultrasonido de baja frecuencia [20].

Es escasa la bibliografía relativa a la aplicación de AE en hormigón reforzado con fibras [21,22], así como también en hormigón sometido a altas temperaturas [23], y prácticamente inexistente en lo que se refiere a hormigón de alta resistencia reforzado con fibras (HSFRC, High Strength Fiber Reinforced Concrete) sometido a altas temperaturas y monitoreados mediante $\mathrm{AE}$.

En este trabajo, se reportan los primeros resultados de una campaña experimental en desarrollo en el Laboratorio de Materiales y Estructuras de la Universidad de Buenos Aires, en colaboración con la Comisión Nacional de Energía Atómica (CNEA). Los mismos corresponden a ensayos de vigas de HSC a flexión para observar el proceso de falla y evaluar sus propiedades residuales luego de la exposición a $600^{\circ} \mathrm{C}$. También se incluye el ensayo de vigas con la misma dosificación pero con la adición de un híbrido de $0,76 \%$ en volumen de macrofibras de acero y $0,1 \%$ en volumen de microfibras de polipropileno con el objeto de estudiar comparativamente la acción de las fibras en el comportamiento del hormigón. Durante todos los ensayos se registró continuamente la AE mediante sensores adheridos a su superficie.

\section{MATERIALES Y MÉTODOS}

El diseño de la mezcla se realizó adoptando una dosificación única para la matriz de hormigón con el objetivo de alcanzar una resistencia a la compresión media en probetas cilíndricas a 28 días de edad mayor a 70 MPa. En la Tabla 1 se detallan los materiales utilizados y su dosificación. Se adoptó una relación agua/materiales cementicios de 0,35. El tamaño máximo nominal de la fracción gruesa fue 9,5 mm y el módulo de finura resultante de la mezcla de agregados 4,3 . Se destaca que todos los materiales empleados son de uso comercial.

Tabla 1: Dosificación de los hormigones $\left[\mathrm{kg} / \mathrm{m}^{3}\right]$.

\begin{tabular}{l|l|l}
\hline COMPONENTE & HSC & HSFRC \\
\hline Cemento CPN50 & 434 & 434 \\
\hline Escoria & 186 & 186 \\
\hline Agua & 217 & 217 \\
\hline Arena fina natural & 283 & 283 \\
\hline Arena de trituración granítica & 512 & 512 \\
\hline Piedra partida granítica & 795 & 795 \\
\hline Fluidificante & 3,1 & 3,1 \\
\hline Macrofibras de acero & - & 60 \\
\hline Microfibras de polipropileno & - & 0,9 \\
\hline
\end{tabular}

Las fibras para hormigón suelen clasificarse en micro y macrofibras según su longitud. Las primeras se emplean para reducir la contracción por secado y prevenir el efecto "spalling" [24], en cambio las macrofibras, al tener mayor anclaje en la masa de hormigón, aumentan la ductilidad y restringen el crecimiento de las macrofisuras [25]. Se utilizaron microfibras de polipropileno monofilamento "FibroMac 12". Sus principales características técnicas son: longitud de fibra $12 \mathrm{~mm}$, diámetro $32 \mu \mathrm{m}$, resistencia a tracción 400-500 $\mathrm{MPa}$, módulo de Young 3500-3900 MPa, temperatura de fusión $160^{\circ} \mathrm{C}$ y peso específico $0,91 \mathrm{~g} / \mathrm{cm}^{3}$. Las macrofibras de acero usadas fueron "Wirand FS3N" (Figura 1). Las propiedades geométricas y mecánicas más sobresalientes de las mismas son: longitud de fibra $33 \mathrm{~mm}$, diámetro $0,75 \mathrm{~mm}$, resistencia a tracción mayor a $1100 \mathrm{MPa}$ y peso específico $7,85 \mathrm{~g} / \mathrm{cm}^{3}$.

Los hormigones fueron ejecutados en una mezcladora de laboratorio, adoptándose la siguiente nomenclatura de acuerdo a la composición del hormigón y a la exposición a temperatura:

-HSC20: hormigón sin fibras de referencia, sin exposición a temperatura elevada.

-HSC600: hormigón sin fibras expuesto a $600^{\circ} \mathrm{C}$ de temperatura.

-HSFRC20: hormigón reforzado con fibras de referencia, sin exposición a temperatura elevada. 
-HSFRC600: hormigón reforzado con fibras sometido a $600^{\circ} \mathrm{C}$ de temperatura.

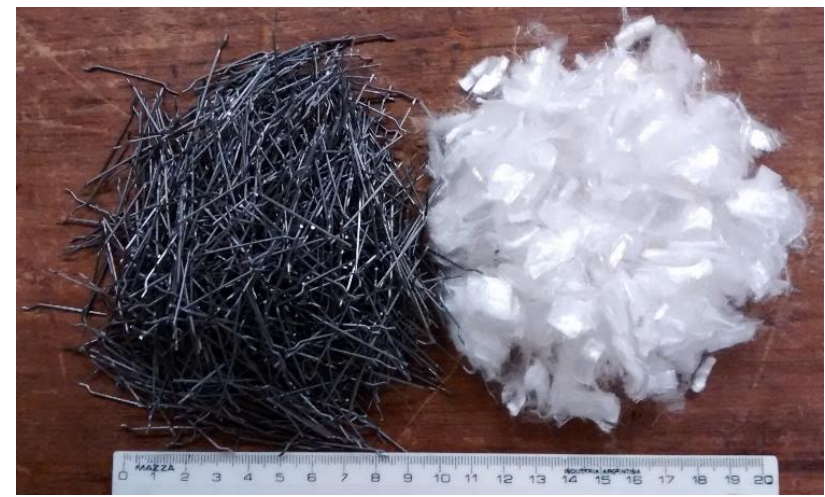

Figura 1: Macrofibras de acero y microfibras de polipropileno utilizadas en HSFRC.

Para cada tipo de hormigón, se moldearon 3 vigas de 150 x 150 x 600 mm y probetas cilíndricas de $100 \mathrm{~mm}$ de diámetro y $200 \mathrm{~mm}$ de altura (Figura 2). Los especímenes fueron desmoldados luego de 24 horas del hormigonado y curados hasta los 28 días de edad sumergidos en agua a $20^{\circ} \mathrm{C}$ de temperatura. Posteriormente, permanecieron almacenados en condiciones de laboratorio para la estabilización de su humedad hasta el tratamiento térmico.

Los especímenes correspondientes a las mezclas HSC600 y HSFRC600 fueron sometidos a $600^{\circ} \mathrm{C}$ de temperatura máxima en un horno eléctrico vertical de 400 x 400 x $700 \mathrm{~mm}$ durante 3 horas (Figura 3). La tasa de calentamiento fue $10^{\circ} \mathrm{C} / \mathrm{min}$. El enfriamiento se realizó lentamente dentro del horno de manera natural y fueron retirados 24 horas después (Figura 4).

Se realizaron tres tipos de ensayos mecánicos: compresión uniaxial, tracción por compresión diametral y flexión de tres puntos (TPB, Three Point Bending Test). Este último es sobre el que se tomaron las mediciones de AE que se reportan en este trabajo y cuyo "setup" se muestra en la Figura 5.

Todos los ensayos se ejecutaron 30 días después del tratamiento térmico en un marco de compresión marca GCTS de 2000 kN de capacidad máxima y con control por lazo cerrado.
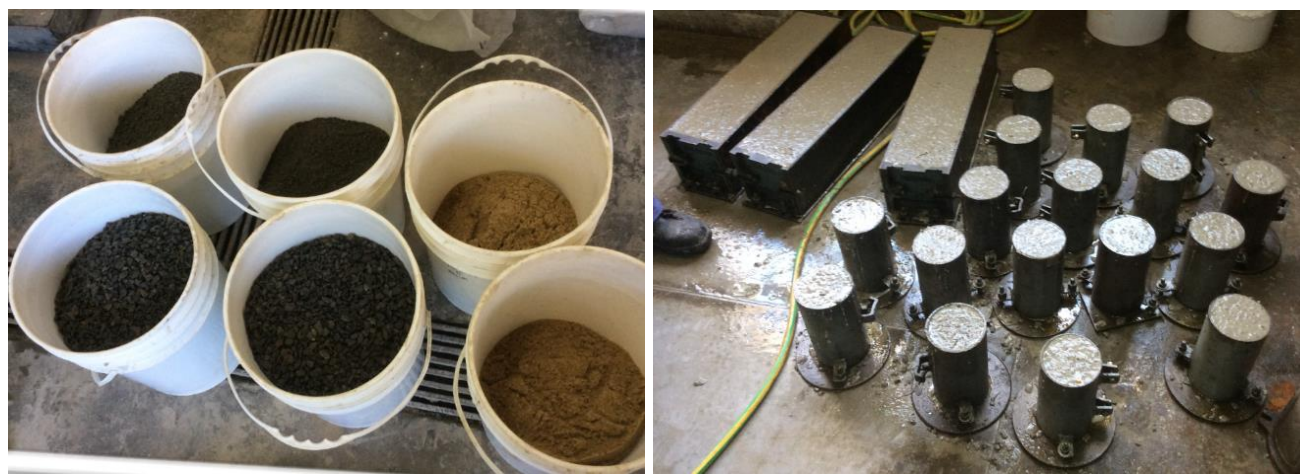

Figura 2: Izquierda: Distintas fracciones de agregados. Derecha: Especímenes en estado fresco. 

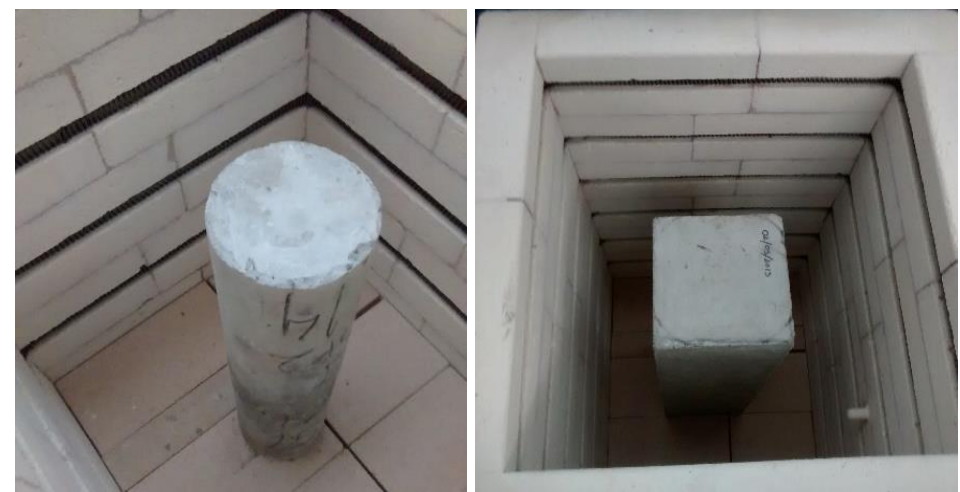

Figura 3: Tratamiento térmico de especímenes a $600^{\circ} \mathrm{C}$ en horno eléctrico.

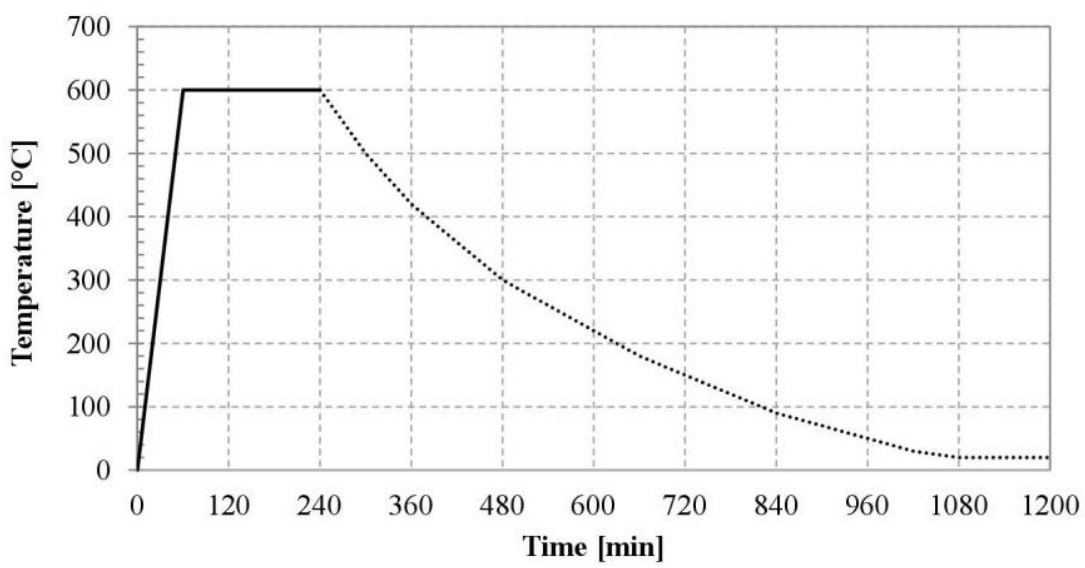

Figura 4: Curva de calentamiento.

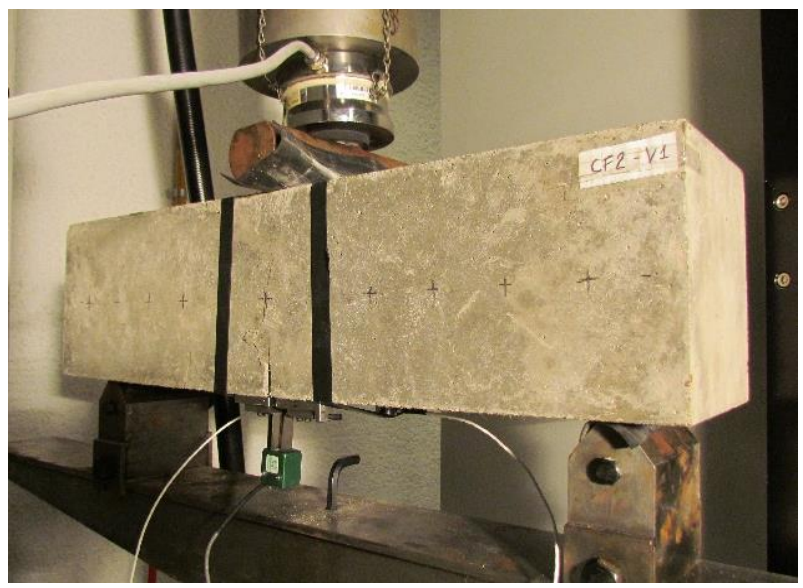

Figura 5: Ensayo de flexión de tres puntos.

El comportamiento a la flexión fue analizado siguiendo, en líneas generales, la recomendación RILEM TC 162-TDF [26]. La luz entre apoyos fue de $500 \mathrm{~mm}$ y la carga se aplicó centrada en coincidencia con la entalla aserrada de $25 \mathrm{~mm}$ de profundidad. El control durante toda la prueba se realizó mediante la abertura del extremo de la entalla (CMOD, Crack Mouth Opening Displacement) a través de un sensor tipo clip gauge marca Epsilon. La velocidad fue 0,05 $\mathrm{mm} / \mathrm{min}$ hasta alcanzar los $0,25 \mathrm{~mm}$ de abertura y luego $0,20 \mathrm{~mm} / \mathrm{min}$ hasta la finalización del ensayo. La medición de CMOD fue corregida según indica la recomendación por la posición del sensor. Con el objeto de obtener una mejor precisión en la medición de la carga, se utilizó una celda de carga externa, marca HBM.

Los ensayos a flexión fueron monitoreados continuamente mediante la técnica de AE. Para ello, se utilizó un equipo marca PAC modelo PCI-2 con dos sensores de la misma marca modelo R15D resonantes en 
$150 \mathrm{kHz}$, acondicionados mediante preamplificadores PAC modelo 2/4/6 con ganancia fijada en $40 \mathrm{~dB}$. Los sensores fueron adheridos a la cara inferior de las vigas según se observa en la Figura 6, centrados y a $5 \mathrm{~cm}$ a cada lado del eje de la entalla. Como acoplante en la interfase sensor-hormigón se usó vaselina sólida. Para reducir el ruido se colocaron aislantes acústicos de caucho en las zonas de apoyo entre acero y hormigón. Previamente al ensayo de cada viga se realizaron pruebas de atenuación de las señales generando fuentes artificiales mediante la rotura de minas de grafito (fuentes de Hsu-Nielsen) en distintos puntos de la superficie. El umbral de medición se estableció en $45 \mathrm{~dB}$ y el rango del filtro de frecuencias entre 20 y $400 \mathrm{kHz}$. Cada forma de onda fue digitalizada y registrada y los parámetros característicos de las mismas como Amplitud, Cuentas, Duración y Signal Strength, fueron calculados por el sistema de AE. En el posprocesamiento de la $\mathrm{AE}$ se filtraron los Hits con amplitud menor a $50 \mathrm{~dB}$ para excluir los rebotes de las señales.
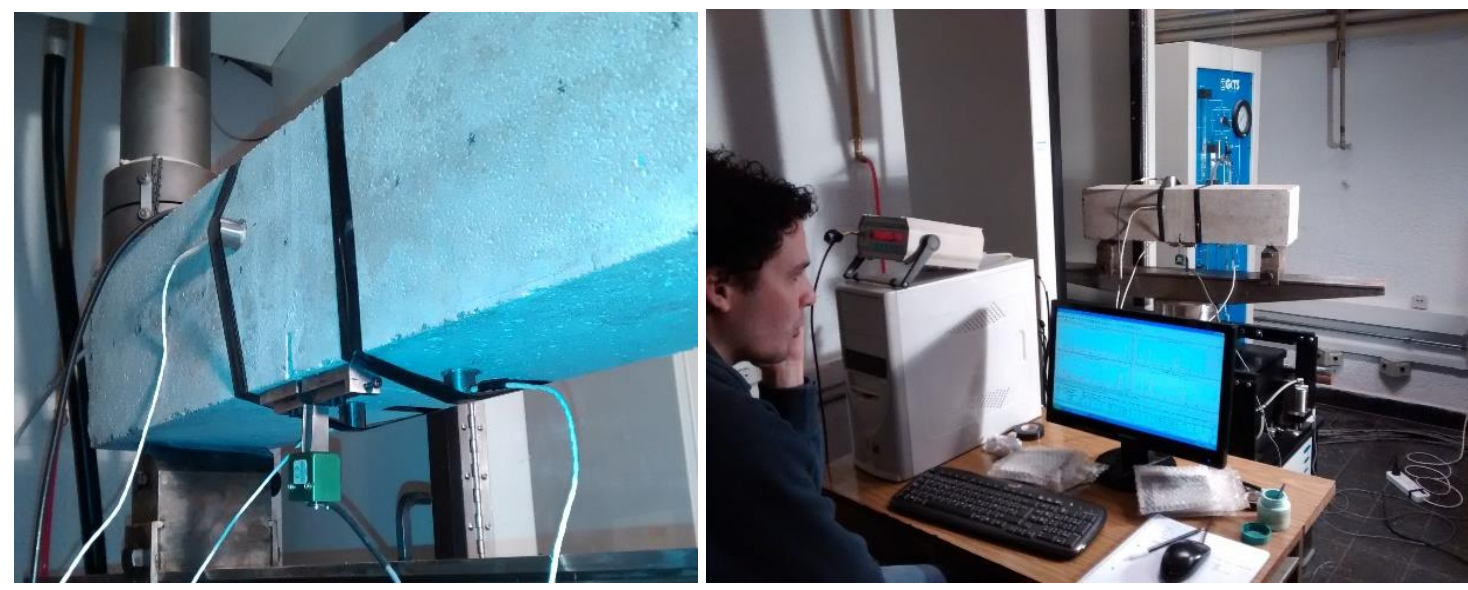

Figura 6: Sensores y equipo de AE.

\section{RESULTADOS Y DISCUSIÓN}

En la Figura 7 se reproducen cortes transversales de los especímenes con fibras HSFRC20 y HSFRC600. En ningún caso ocurrió "spalling", no obstante, se observaron numerosas fisuras en la superficie de las muestras tratadas. También ocurrió un significativo cambio de coloración del hormigón, tendiendo a un gris más claro en la matriz y al blanco y rosado en los agregados gruesos.

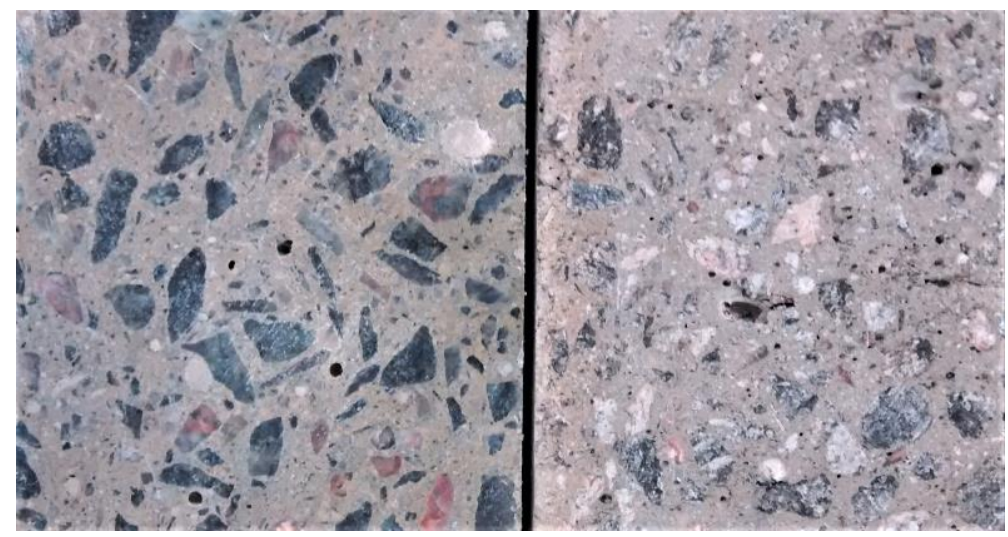

Figura 7: Cortes transversales de especímenes reforzados con fibras. Izquierda: HSFRC20. Derecha: HSFRC600.

Los resultados de resistencia a compresión uniaxial se encuentran representados en la Figura 8. La adición de las fibras generó una disminución en la resistencia a compresión probablemente debido a la formación de zonas de interfase matriz-fibras de mayor porosidad que debilitaron la respuesta del material compuesto. Este fenómeno se vio agravado por tratarse de un hormigón de muy baja relación agua-cemento.

En la Figura 9 se observan los resultados de resistencia a compresión uniaxial expresados en términos de resistencia residual relativa. Se aprecia una significativa disminución por efecto de la temperatura y de proporción casi idéntica en ambos casos: 75\% en el HSFRC600 y 77\% en el HSC600. Por lo tanto, la adición de fibras no mejoró la resistencia residual pico a compresión luego de ser sometido a $600^{\circ} \mathrm{C}$ de temperatura. 
En las Figuras 10 y 11 se presenta la resistencia a tracción por compresión diametral para ambos hormigones, en términos absolutos y relativos, respectivamente. Se aprecia que la resistencia a tracción indirecta resultó mayor para el caso del hormigón con fibras. También puede observarse que para el caso de hormigón con fibras dicha resistencia fue considerablemente menos degradada luego del tratamiento térmico, conservando un 40\% de la resistencia del HSFRC20 en contraste con el 18\% para el hormigón sin fibras.

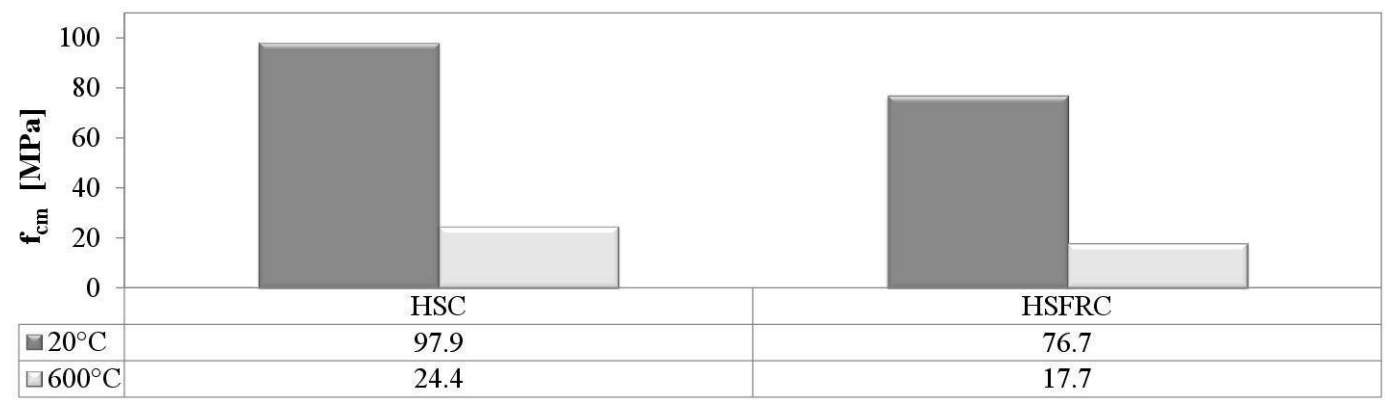

Figura 8: Resistencia a compresión uniaxial.

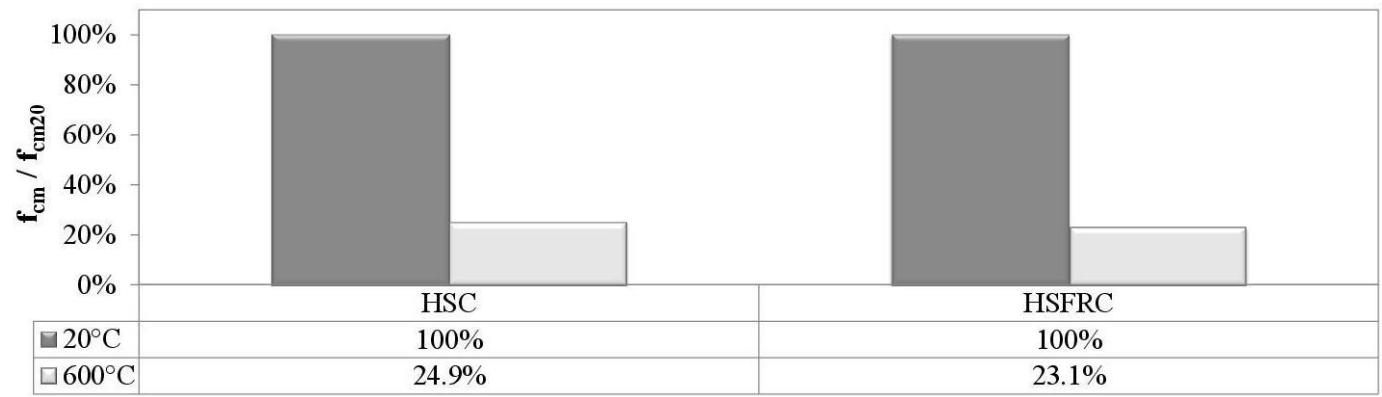

Figura 9: Resistencia a compresión uniaxial relativa.

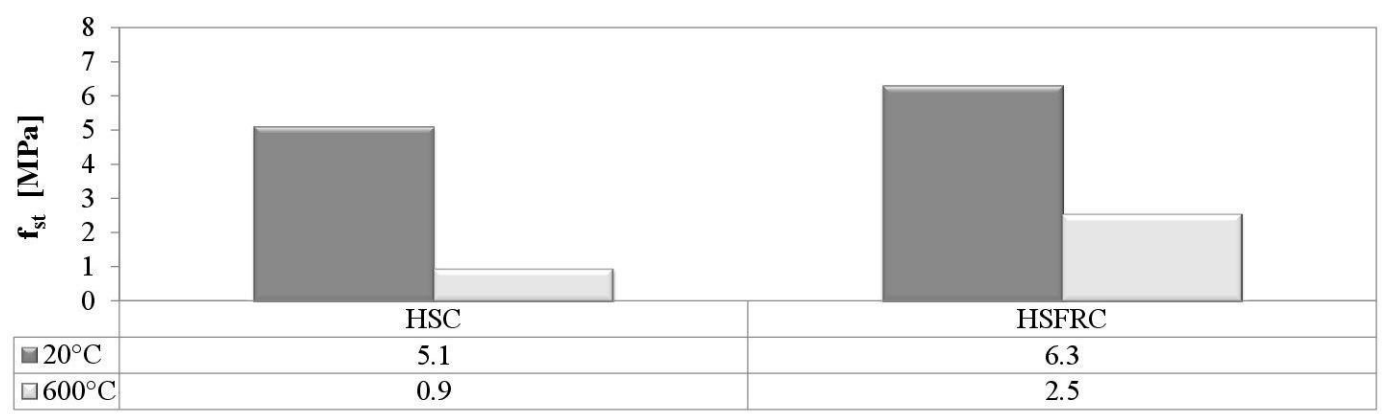

Figura 10: Resistencia a tracción por compresión diametral.

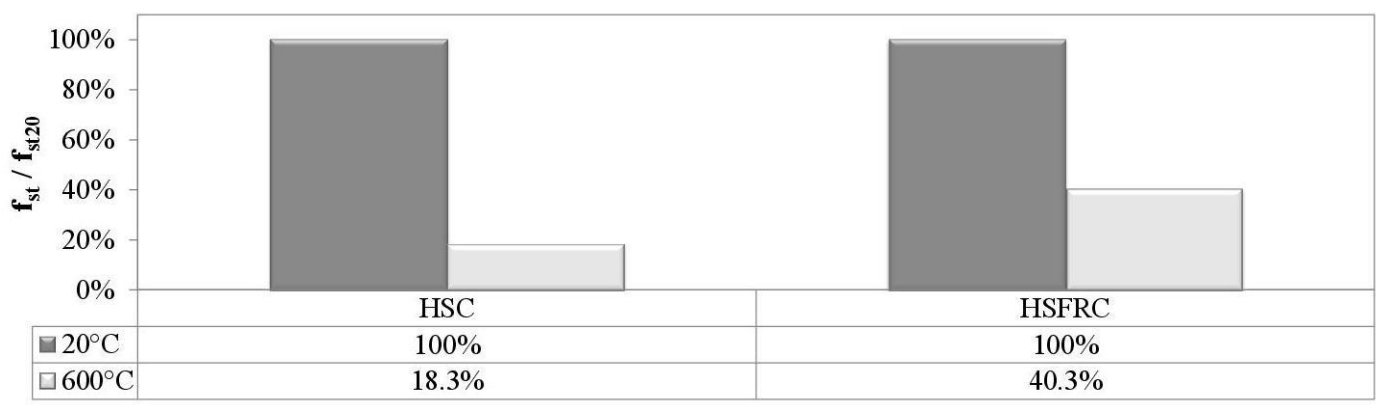

Figura 11: Resistencia a tracción por compresión diametral relativa.

En las Figuras 12 y 13 se muestran las curvas de carga en función de CMOD para los casos tempera- 
tura ambiente y luego del tratamiento térmico a $600^{\circ} \mathrm{C}$, respectivamente. En la primera se puede apreciar el importante incremento en la capacidad de absorción de energía que exhibe el HSFRC20 en la etapa posfisuración respecto al HSC20. Como era de esperarse, la carga pico inicial se vio muy poco afectada por la presencia de las fibras debido a que las mismas entran en carga principalmente luego de fisurarse la matriz cementicia. También se observa que para el HSC20 la CMOD máxima alcanzada fue menor a 1,5 mm, mientras que en el caso con fibras, las vigas aún tenían capacidad de carga al darse por finalizado el ensayo.
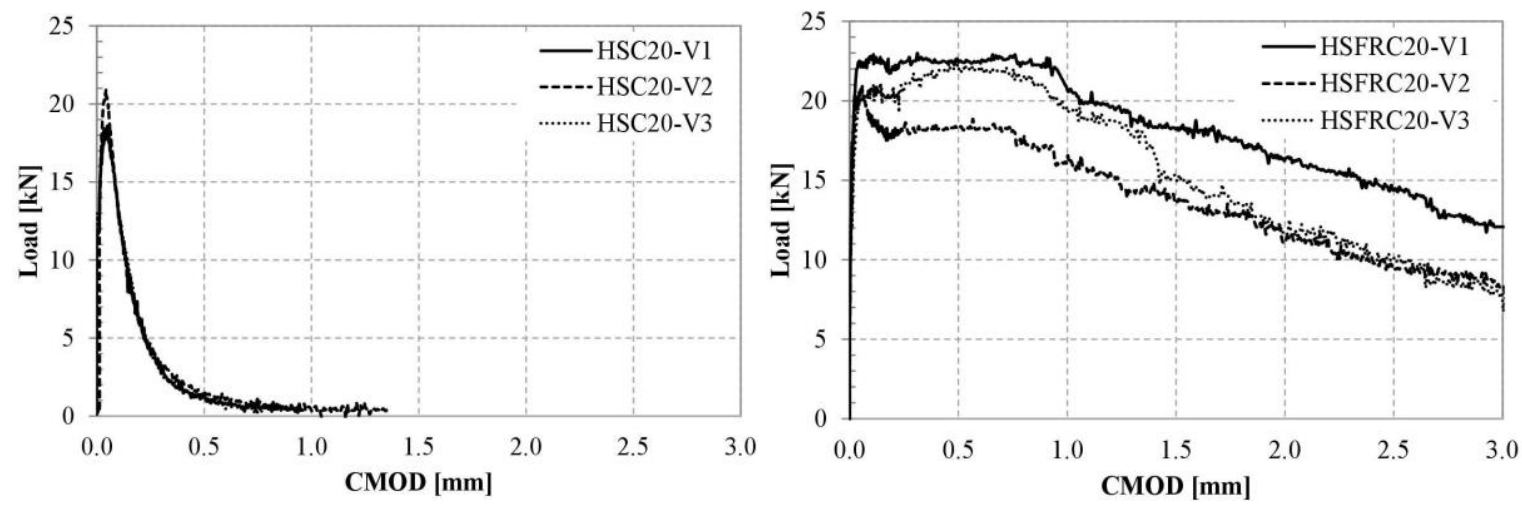

Figura 12: Curvas de Carga vs. CMOD. Vigas a temperatura ambiente.
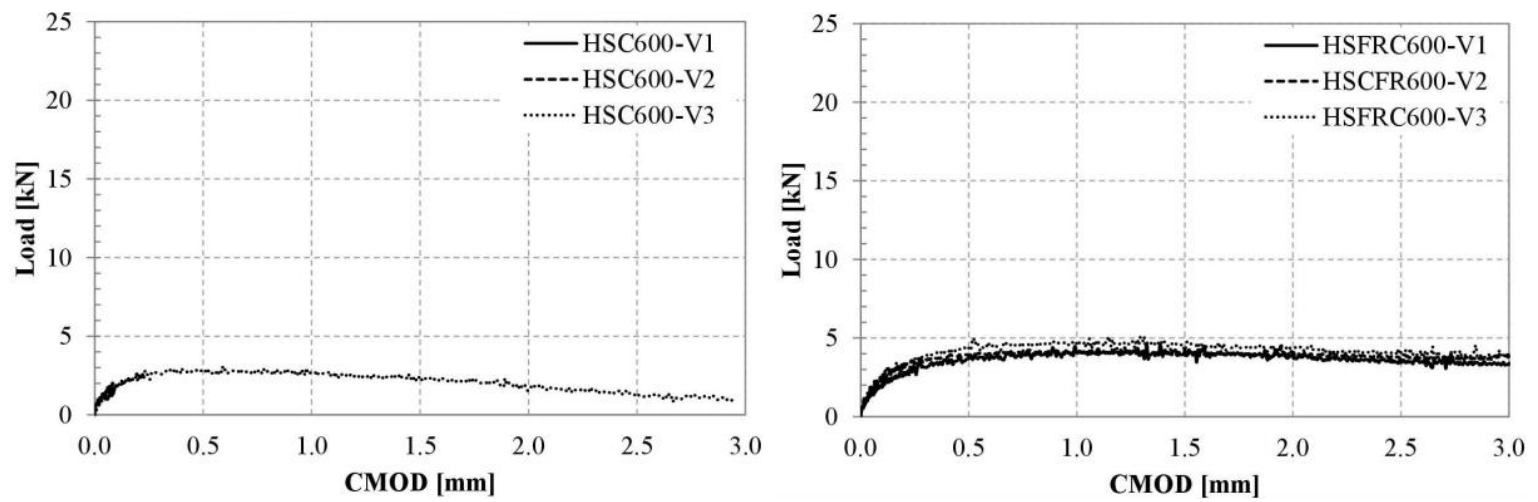

Figura 13: Curvas de Carga vs. CMOD. Vigas tratadas a $600^{\circ} \mathrm{C}$ de temperatura.

En la Figura 13, y en comparación con la Figura 12, se aprecia una importante degradación del comportamiento resistente por la acción de la temperatura. En el caso del HSC600, dos de los tres prismas fallaron abruptamente cuando alcanzaban la carga máxima. Las fracturas se produjeron próximas a las entallas a través de fisuras que se habían generado durante el tratamiento térmico, en ambos casos para CMOD menores a $0,25 \mathrm{~mm}$, resultando en una muy baja capacidad de absorción de energía. Sólo un ensayo de HSC600 pudo desarrollarse hasta los $3 \mathrm{~mm}$ de abertura, en marcado contraste con el comportamiento original de ese hormigón. El módulo resistente a la flexión, obtenido a partir de la carga máxima y las características geométricas de las vigas y del ensayo, se redujo al 13\% del HSC20. Para el HSFRC600, todos los especímenes se comportaron de manera similar garantizando la capacidad portante durante todo el ensayo. En este caso, el módulo resistente se redujo al 20\% del HSFRC20. Por lo tanto, la adición de fibras en el hormigón brindó una mayor confiabilidad en la respuesta estructural frente a la acción de altas temperaturas que el hormigón simple.

En la Figura 14 se muestra de manera superpuesta la respuesta mecánica a flexión y la AE acumulada para los hormigones HSC20 y HSFRC20. Similarmente, en la Figura 17 se reproduce para los hormigones luego del tratamiento térmico. En ambos casos la AE crece más rápidamente cuando no hay fibras en el hormigón, alcanzando hacia el final del ensayo cantidades acumuladas similares. Para distintos valores de CMOD, la AE acumulada es mayor en el HSC. Este fenómeno se debió a que al aplicarse una dada CMOD la fisura encuentra menor resistencia a su crecimiento en el hormigón simple que en el hormigón reforzado con fibras. Por lo tanto, la fisura alcanzó mayor altura en el espécimen implicando un mayor nivel de daño temprano en la viga. Una vez fracturada prácticamente la totalidad de la sección, se advierte que va disminuyendo la pendiente de la curva de AE acumulada. No obstante, se siguió registrando AE debido a la fricción pro- 
ducto de la intertrabazón de los agregados, la interacción entre las nuevas superficies de la matriz y el deslizamiento de las fibras. Este comportamiento de AE es muy diferente del típicamente observado en ensayos de hormigón sin fibras donde el control se realiza a través del desplazamiento del pistón [21-23]. En tales ensayos se produce la rotura abrupta del espécimen al alcanzarse la carga máxima, generando una importante cantidad de AE de manera instantánea.

Tomando en cuenta que los estados límites de servicio para el diseño de las estructuras de hormigón limitan las aberturas de fisuras como máximo a $0,4 \mathrm{~mm}$, para el entorno de ese valor, el nivel de daño caracterizado por la AE acumulada, es significativamente menor para el HSFRC.

En las Figuras 15 y 16 se presentan secuencias fotográficas del crecimiento de la fisuración superficial de especímenes de HSC20 y HSFRC20, y en las Figuras 18 y 19 las correspondientes a HSC600 y HSFRC600.

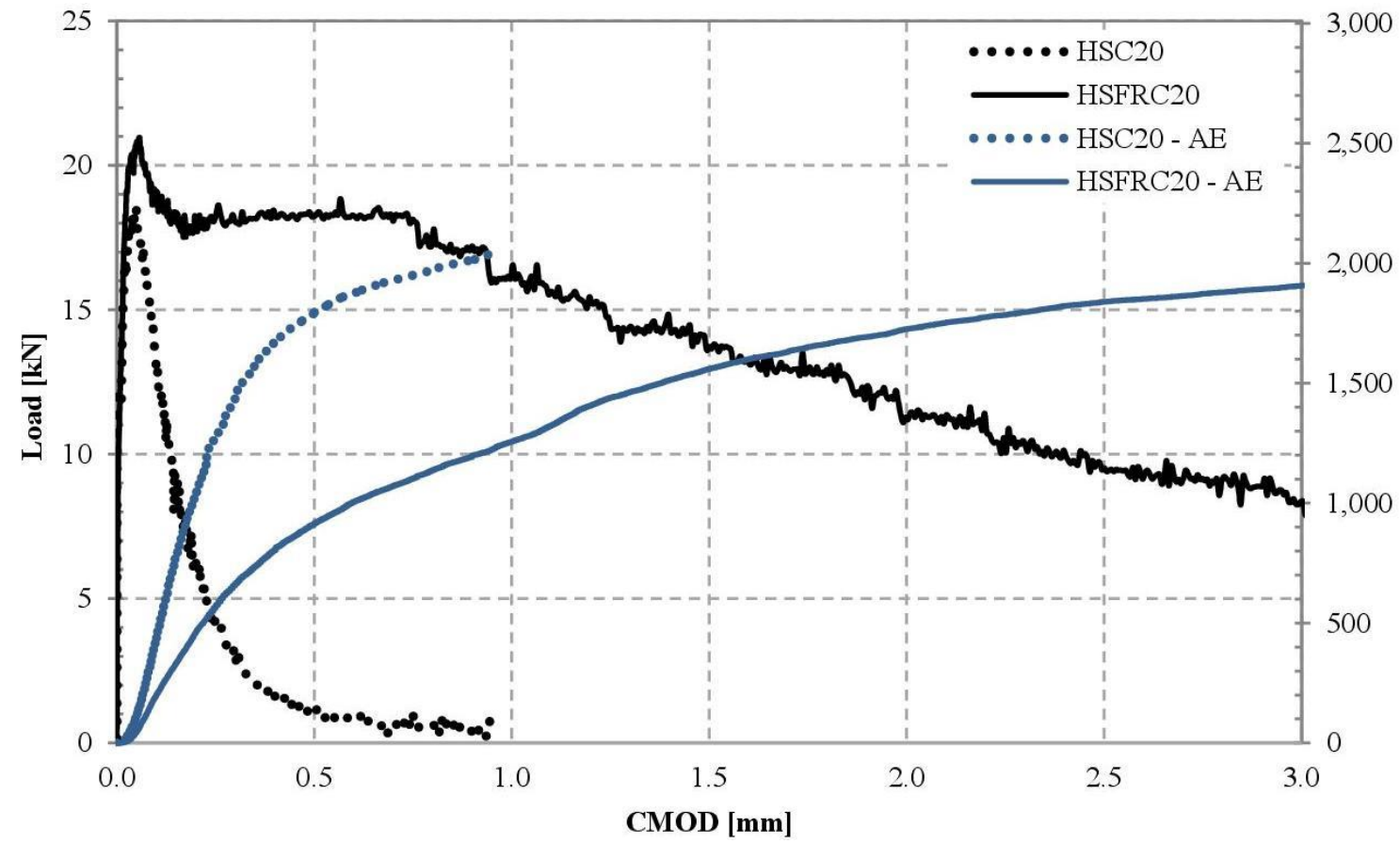

Figura 14: Curvas de Carga y AE Acumulada vs. CMOD. Vigas a temperatura ambiente.

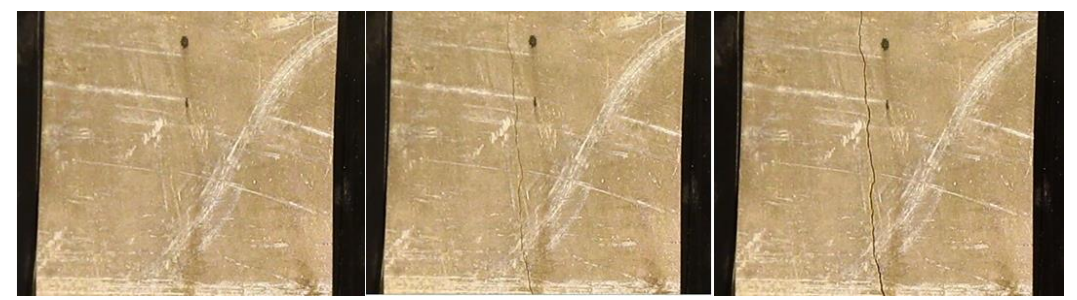

Figura 15: Secuencia fotográfica HSC20 correspondiente a: $\mathrm{CMOD}=0,05 \mathrm{~mm} ; \mathrm{CMOD}=0,5 \mathrm{~mm}$ y CMOD $=1,0 \mathrm{~mm}$.

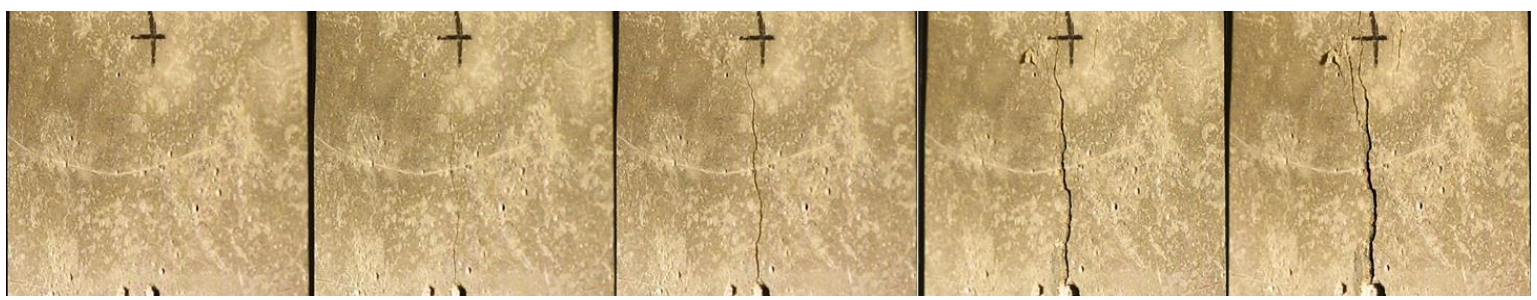

Figura 16: Secuencia fotográfica HSFRC20 correspondiente a: $\mathrm{CMOD}=0,05 \mathrm{~mm} ; \mathrm{CMOD}=0,5 \mathrm{~mm} ; \mathrm{CMOD}=1,0$ $\mathrm{mm} ; \mathrm{CMOD}=2,0 \mathrm{~mm}$ y $\mathrm{CMOD}=3,0 \mathrm{~mm}$. 


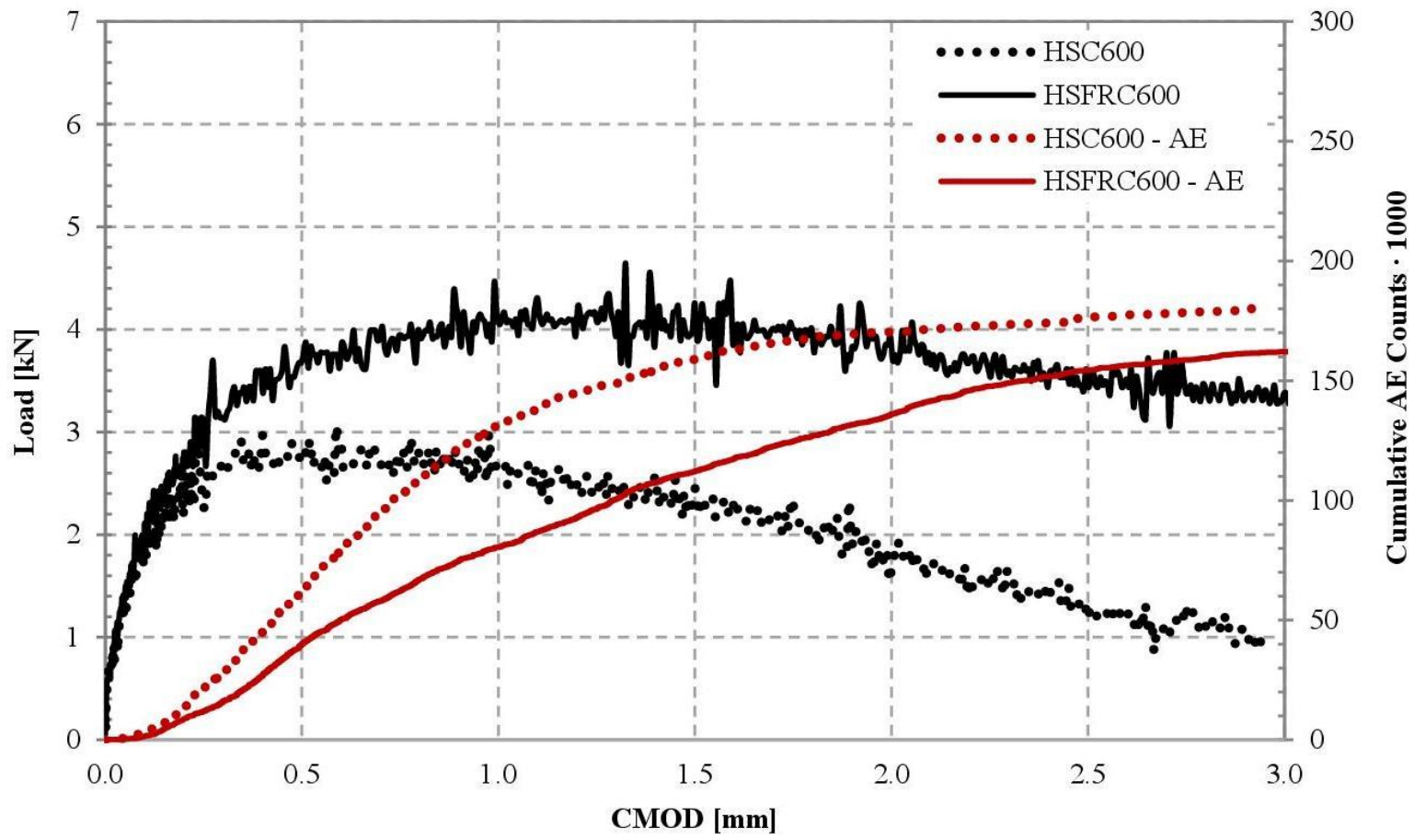

Figura 17: Curvas de Carga y AE Acumulada vs. CMOD. Vigas tratadas a $600^{\circ} \mathrm{C}$ de temperatura.

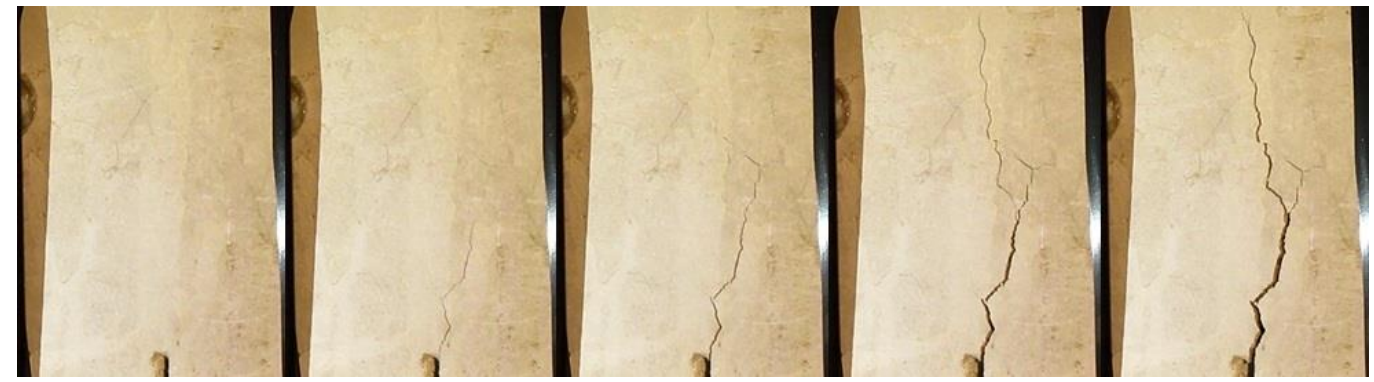

Figura 18: Secuencia fotográfica HSC600 correspondientes a: $\mathrm{CMOD}=0,05 \mathrm{~mm} ; \mathrm{CMOD}=0,5 \mathrm{~mm} ; \mathrm{CMOD}=1,0 \mathrm{~mm}$; $\mathrm{CMOD}=2,0 \mathrm{~mm}$ y $\mathrm{CMOD}=3,0 \mathrm{~mm}$.

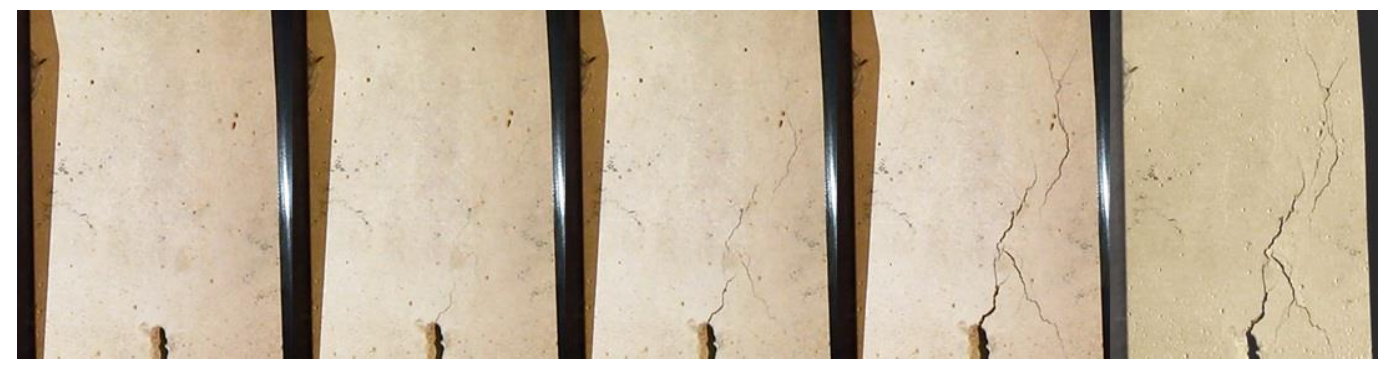

Figura 19: Secuencia fotográfica HSFRC600 correspondientes a: $\mathrm{CMOD}=0,05 \mathrm{~mm} ; \mathrm{CMOD}=0,5 \mathrm{~mm} ; \mathrm{CMOD}=1,0$ $\mathrm{mm} ; \mathrm{CMOD}=2,0 \mathrm{~mm}$ y $\mathrm{CMOD}=3,0 \mathrm{~mm}$.

En el caso de los hormigones sometidos a $600^{\circ} \mathrm{C}$, la cantidad de emisión registrada por los sensores fue considerablemente menor, así como también la amplitud máxima de las señales. Las fisuras preexistentes producidas por la acción de la temperatura dificultan la transmisión de las ondas en el material, incrementando considerablemente la atenuación de las señales.

La fuerte relación entre la AE y la fisuración puede apreciarse en las Figuras 20 y 21, en las que se representa la variación temporal de la carga vertical y la $\mathrm{AE}$ acumulada de las vigas para los casos sin y con tratamiento térmico, respectivamente. Como se mencionó anteriormente, durante la ejecución de los ensayos se realizó un aumento de velocidad de abertura a partir de CMOD igual a 0,25 mm, correspondiente a 300 
segundos del comienzo del ensayo. En ese momento y para todos los ensayos, se puede ver un fuerte incremento de la pendiente de la AE acumulada, así como también se observó un aumento en la actividad de todos los parámetros de AE. En particular, se advierte la baja cantidad de emisiones en el comienzo de los ensayos de los especímenes sometidos a $600^{\circ} \mathrm{C}$. Durante el tratamiento térmico se degradó el material generándose múltiples fisuras y, por lo tanto, ocurrió una importante liberación irreversible de energía interna que se manifiesta como AE [27]. Luego, al ensayar las vigas dicha energía ya no se encuentra disponible, siendo necesario el transcurso de un lapso de tiempo para activar las fisuras preexistentes.

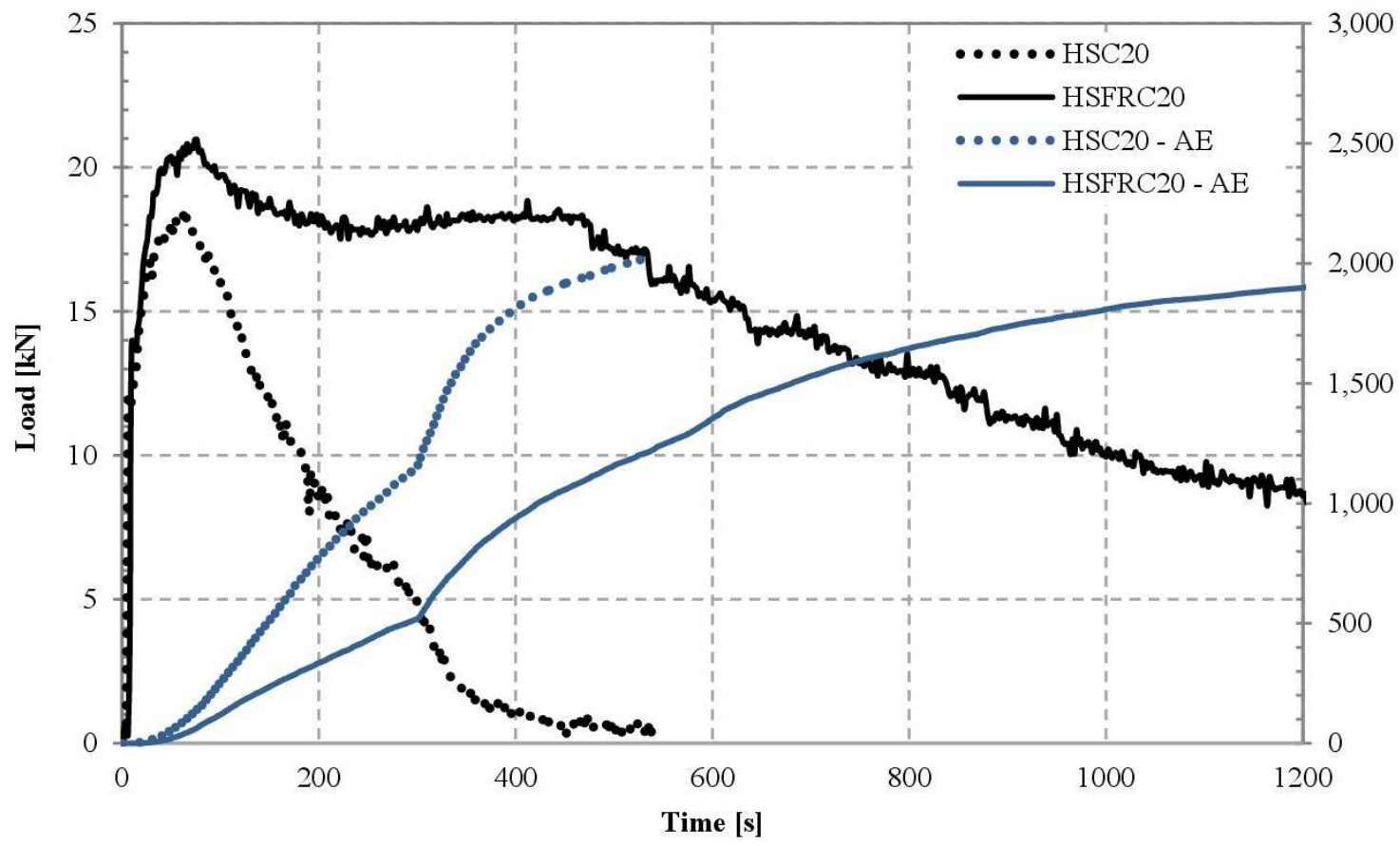

Figura 20: Curvas de Carga y AE Acumulada vs. Tiempo. Vigas a temperatura ambiente.

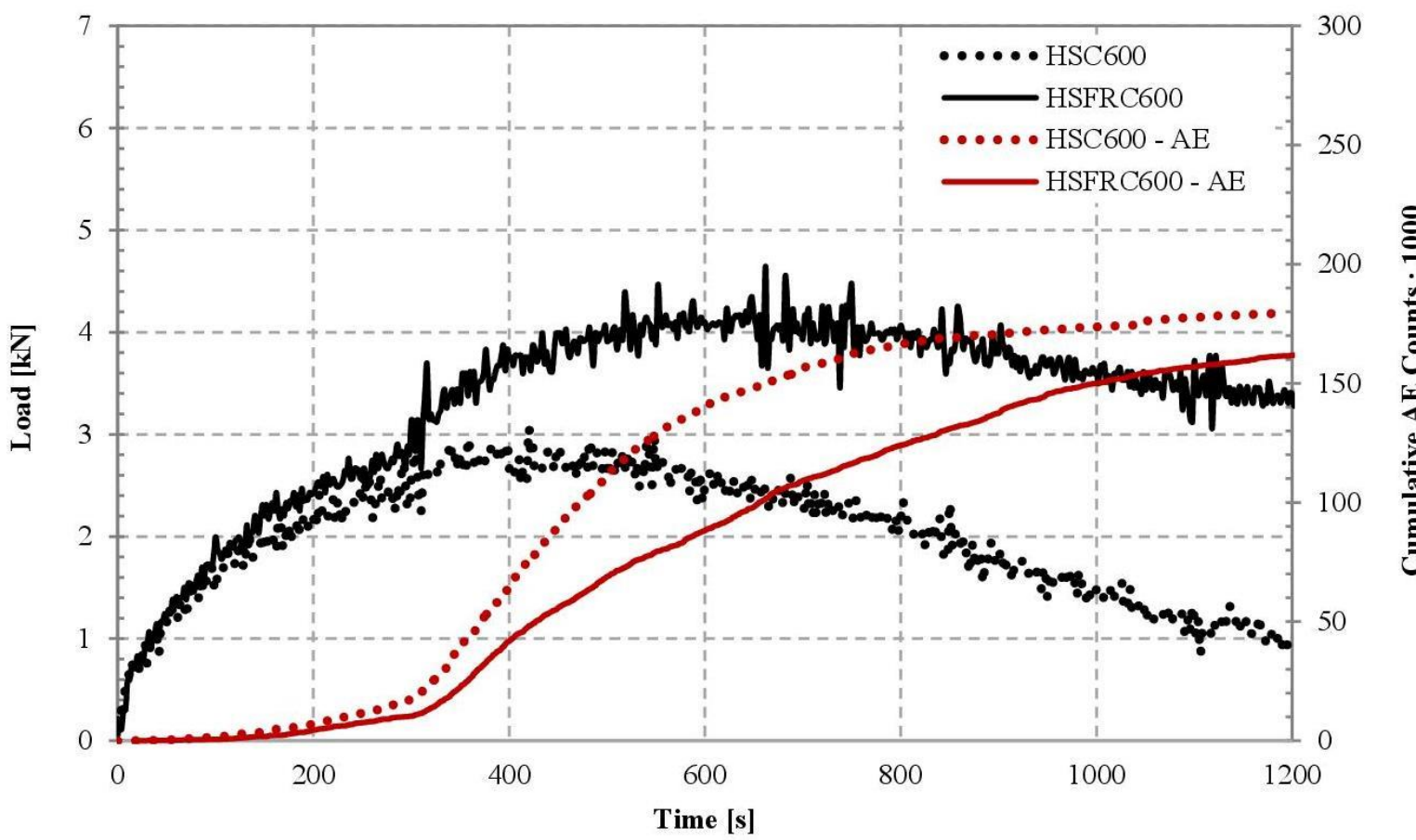

Figura 21: Curvas de Carga y AE Acumulada vs. Tiempo. Vigas tratadas a $600^{\circ} \mathrm{C}$ de temperatura. 


\section{CONCLUSIONES}

En este trabajo se han presentado y discutido los primeros resultados de una campaña experimental en desarrollo relativa al comportamiento del hormigón frente a la acción de temperaturas elevadas. Se estudiaron hormigones de alta resistencia con y sin adición de un híbrido de fibras de acero y polipropileno. Algunos especímenes fueron expuestos a la temperatura máxima de $600^{\circ} \mathrm{C}$ durante 3 horas y posteriormente ensayados para conocer su respuesta mecánica residual en comparación con los mismos hormigones sin tratamiento térmico. En particular, se utilizó la técnica de la AE durante los ensayos a flexión en tres puntos para monitorear el desarrollo de la fractura.

Basándose en los resultados obtenidos, se remarcan las siguientes observaciones desde el punto de vista del comportamiento resistente:

- El hormigón reforzado con fibras sufrió una degradación en su resistencia a la compresión relativa similar a la del hormigón simple, en ambos casos del orden del 75\% luego de ser expuestos a $600^{\circ} \mathrm{C}$. Por el contrario, se observaron importantes incrementos de resistencia residual a la tracción por compresión diametral y en su comportamiento a la flexión.

- La resistencia flexional pico con y sin fibras fue más afectada por el efecto de la temperatura que la resistencia a la compresión.

- Se observó una marcada no linealidad en la respuesta flexional debido a la acción de la temperatura, como consecuencia de la degradación y pérdida de rigidez de la matriz.

- El hormigón reforzado con fibras siguió teniendo capacidad de absorción de energía luego del tratamiento térmico, alcanzado valores de CMOD de $3 \mathrm{~mm}$.

Respecto al monitoreo mediante la técnica de $\mathrm{AE}$ en los ensayos a flexión, se destaca lo siguiente:

- La pendiente inicial de la curva de AE acumulada, tanto en función del tiempo como de CMOD, está en relación inversa a la ductilidad de la falla. Cuanto mayor es la pendiente, menor es la ductilidad.

- La AE acumulada en las vigas sin fibras, tanto en función del tiempo como de CMOD, con y sin tratamiento térmico, muestra una mayor pendiente que la del hormigón con fibras, demostrando la efectividad de las fibras para restringir la propagación de las fisuras.

- La degradación térmica también quedó de manifiesto en las características de la AE registrada. Las macrofisuras por flexión se desarrollan a partir de las fisuras causadas por la temperatura, generando menor cantidad de eventos de AE y de menor amplitud.

- La AE es fuertemente sensible al proceso de fisuración del hormigón, lo cual quedó claramente comprobado en los ensayos realizados controlados por velocidad de abertura de fisura constante. En virtud de ello, es una técnica de monitoreo remoto promisoria para estructuras de hormigón por su estrecha relación con el nivel de daño.

\section{AGRADECIMIENTOS}

Se agradece al Sr. Luciano Sambataro por su valiosa colaboración en la preparación y ejecución de los ensayos y al Dr. Luis Fernandez Luco, Director del Laboratorio de Materiales y Estructuras de la FIUBA.

\section{BIBLIOGRAFÍA}

[1] CASTILLO, C., DURRANI, A.J., "Effect of Transient High Temperature on High-Strength Concrete", ACI Materials Journal, v. 87, n. 1, pp. 47-53, Jan.-Feb. 1990.

[2] ACI-544.1-96: "State-of-the-art report on fiber reinforced concrete". Reported by ACI Committee 544, American Concrete Institute, 1996.

[3] HAN, C-G, HWANG, Y-S, YANG, S-H, GOWRIPALAN N., "Performance of Spalling Resistance of High Performance Concrete with Polypropylene Fibers Contents and Lateral Confinement", Cement and Concrete Research, v. 35, pp. 1747-1753, 2005.

[4] RILEM TC 200-HTC: "Mechanical concrete properties at high temperatures - modelling and applications. Part 1: Introduction - General presentation", Materials and Structures, v. 35, pp. 579-582, 2002.

[5] CHANG, Y. F., CHEN, Y. H., SHEU, M. S., et al., "Residual stress-strain relationship for concrete after exposure to high temperatures", Cement Concrete Research, v. 36, pp. 1999-2005, 2006. 
[6] DI MAIO, A., HIRSCHI, F., GIACCIO, G., et al., "Hormigones expuestos a altas temperaturas: evaluación de la permeabilidad y otras propiedades físicas", In: Anales SAM, pp. 204-209, 1999.

[7] LEE, J., XI, Y., WILLAM, K., "Properties of concrete after high-temperature heating and cooling", ACI Materials Journal, v. 105, pp. 334-341, 2008.

[8] HARMATHY, T., "Thermal properties of concrete at elevated temperature", Journal of Materials, v. 5, pp. 47-74, 1970.

[9] KHOURY, G. A., "Effect of fire on concrete and concrete structures", Prog. Struct. Engng Mater., v. 2, n. 4, pp. 429-447, 2000.

[10] MARECHAL, J. C., "Variations in the modulus of elasticity and Poisson's ratio with temperature", $A C I$ Special Publication 34,n. 1, pp. 495-503, 1972.

[11] JANOTKA, I., BAGEL, L., "Pore structures, permeabilities, and compressive strengths of concrete at temperatures up to $800^{\circ} \mathrm{C}$ ", ACI Materials Journal, v. 100, pp. 196-200, 2002.

[12] PHAN, L. T., CARINO, N. J., "Effects of test conditions and mixture proportions on behavior of highstrength concrete exposed to high temperatures", ACI Materials Journal, v. 99, pp. 54-66, 2002.

[13] BAKER, G., "The effect of exposure to elevated temperatures on the fracture energy of plain concrete", Materials and Structures, v. 39, pp. 47-61, 1996.

[14] NIELSEN, C. V., BICANIC, N., "Residual fracture energy of high-performance and normal concrete subject to high temperatures", Materials and Structures, v. 36, pp. 545-521, 2003.

[15] OHNO, K., UJI, K., ATSUSHI, U., OHTSU, M., "Fracture process zone in notched concrete beam under three-point bending by acoustic emission", Construction and Building Materials, v. 67, pp. 139-145, 2014.

[16] SAGAR, R.V., PRASAD, B.K.R., "An experimental study on acoustic emission energy as a quantitative measure of size independent specific fracture energy of concrete beams", Construction and Building Materials, v. 25, pp. 2349-2357, 2011.

[17] DAI, Q., NG, K., ZHOU, J., KREIGER, E., et al., "Damage investigation of single-edge notched beam tests with normal strength concrete and ultra high performance concrete specimens using acoustic emission techniques", Construction and Building Materials, v. 31, pp. 231-242, 2012.

[18] SALIBA, J., LOUKILI, A., REGOIN, J.P., et al., "Experimental analysis of crack evolution in concrete by the acoustic emission technique", Frattura e Integritá Strutturale, v. 34, pp. 300-308, 2015.

[19] ASTM E 1316-04: "Standard Terminology for Nondestructive Testing", Books of Standards, ASTM International, W. Conshohocken, PA, USA, 2004.

[20] GROSSE, C.U., OHTSU, M., Acoustic emission testing, Berlin Heidelberg, Springer-Verlag, 2008.

[21] SOULIOTI, D., BARKOULA, N.M., PAIPETIS, A.S., et al., "Acoustic emission behavior of steel fibre reinforced concrete under bending", Construction and Building Materials, v. 23, pp. 3532-3536, 2009.

[22] AGGELIS, D.G., SOULIOTI, D., SAPOURIDIS N., et al., "Acoustic emission characterization of the fracture process in fibre reinforced concrete", Construction and Building Materials, v. 25, pp. 4126-4131, 2011.

[23] GENG, J., SUN, Q., ZHANG, W., et al., "Effect of high temperature on mechanical and acoustic emission properties of calcareous-aggregate concrete", Applied Thermal Engineering, v. 106, pp. 1200-1208, 2016.

[24] BEHNOOD, A., GHANDEHARI, M., "Comparison of compressive and splitting tensile strength of high-strength concrete with and without polypropylene fibers heated to high temperatures", Fire Safety Journal, v. 44, pp. 1015-1022, 2009.

[25] CAGGIANO, A., XARGAY, H., FOLINO, P., et al., "Experimental and numerical characterization of the bond behavior of steel fibers recovered from waste tires embedded in cementitious matrices", Cement \& Concrete Composites, v. 62, pp. 146-155, 2015.

[26] RILEM TC 162-TDF: "Test and design methods for steel fibre reinforced concrete - Bending test, final recommendation", Materials and Structures, v. 40, pp. 841-853, 2007.

[27] GROSSE, C., OŽBOLT, J., RITCHER, R., et al., "Acoustic emission analysis and thermo-hygromechanical model for concrete exposed to fire", J. Acoustic Emission, v. 28, pp. 188-203, 2010. 